\title{
Associações entre Estilos Parentais, Interesses e Indecisão Profissional em Estudantes do Ensino Médio
}

\author{
Camélia Santina Murgo ${ }^{1}$ \\ Leonardo de Oliveira Barros ${ }^{2}$ \\ Bárbara Cristina Soares Sena \\ ${ }^{1}$ Universidade do Oeste Paulista, Presidente Prudente, SP \\ ${ }^{2}$ Universidade São Francisco, Campinas, SP
}

\begin{abstract}
Resumo
Este estudo objetivou verificar as relações entre estilos parentais, interesses profissionais e indecisão de adolescentes. Participaram desta pesquisa 472 estudantes de ensino médio de escolas públicas do interior de São Paulo, sendo 66\% do sexo feminino e com idade média de 16,55 anos. Foram aplicados o Questionário de Busca Autodirigida (SDS), a Escala de Responsividade e Exigência (ERE) e o Inventário de Levantamento das Dificuldades de Decisão Profissional (IDDP). Os resultados indicaram que quanto menor a dificuldade de decisão em função do prestígio profissional e sucesso financeiro, maiores os interesses empreendedores e que, quanto maior esse tipo de dificuldade, maior a percepção de mães como exigentes e pais como responsivos e exigentes. Verificou-se que adolescentes com maiores interesses realistas e empreendedores tendem a perceber a figura paterna com maior responsividade. Sugerem-se investigações que ampliem a compreensão dos achados e novas discussões sobre o tema na área da Orientação Profissional e de Carreira.

Palavras-chave: orientação vocacional, adolescência, indecisão profissional, estilo parental, avaliação psicológica
\end{abstract}

\section{Associations between Parenting Styles, Interests and Professional Indecision in High School Students}

\begin{abstract}
This study aimed to verify the relationships between parental styles, professional interests and indecision of adolescents. A total of 472 high school students from public schools in the country of the state of São Paulo, Brazil, participated in this study, 66\% female and with a mean age of 16.55 years. The Self-Directed Search (SDS), the Responsibility and Requirement Scale (ERE) and the Professional Decision Difficulties Survey Inventory (IDDP) were applied. The results indicated that the lower the difficulty of decision due to professional prestige and financial success, the greater the entrepreneurial interests; and the greater this type of difficulty, the greater the perception of mothers as demanding and fathers as responsive and demanding. It was found that adolescents with greater realistic and entrepreneurial interests tend to perceive the father figure with greater responsiveness. We suggest further research that broadens the understanding of the findings and new discussions on the subject in the field of Professional and Career Guidance.

Keywords: Vocational guidance; adolescence; professional indecision; parenting style; psychological evaluation
\end{abstract}

\section{Asociaciones entre Estilos Parentales, intereses e indecisión profesional en estudiantes de Enseñanza Secundaria}

\section{Resumen}

Este estudio tuvo como objetivo verificar las asociaciones entre los estilos parentales, intereses profesionales e indecisión de los adolescentes. Participaron de esta investigación 472 estudiantes de Enseñanza Secundaria de escuelas públicas del interior de San Pablo, siendo 66\% de sexo femenino y con un promedio de edad de 16,55 años. Se aplicó Cuestionario de Búsqueda Auto-dirigida (SDS), Escala de Sensibilidad y Exigencia (ERE) e Inventario de Levantamiento de las Dificultades de Decisión Profesional (IDDP). Los resultados indicaron que cuanto menor es la dificultad de decisión en función del prestigio profesional y suceso financiero, mayores son los intereses emprendedores, y que cuanto mayor es la dificultad, mayor es la percepción de las madres como exigentes y de los padres como sensibles y exigentes. Se constató que los adolescentes con mayores intereses realistas y emprendedores tienden a percibir la figura paterna con más sensibilidad. Se sugieren investigaciones que amplíen la comprensión de los resultados y nuevas discusiones sobre el tema en el área de la Orientación Profesional y de Carrera.

Palabras-clave: orientación vocacional, adolescencia, indecisión profesional; estilo parental; evaluación psicológica

\section{Introdução}

Durante a adolescência surgem as primeiras preocupações com a tomada de decisão profissional, existindo a necessidade de orientações que ampliem a condição do adolescente de alcançar clareza sobre suas habilidades, metas e interesses profissionais. Tais direcionamentos podem surgir do contexto social do adolescente e, em especial, do âmbito familiar que é responsável por transmitir valores que influenciam na tomada de decisão (Bacal, Magalhães, \& Féres-Carneiro, 2014). Além da contribuição familiar, a Orientação 
Profissional e de Carreira (OPC) tende a contribuir ao compreender as necessidades de realização e autoestima, bem como as influências das diversas mudanças sociais, econômicas, políticas (Duarte, 2009) e familiares que auxiliam na classificação das habilidades e dos interesses dos orientandos (Sartori, Noronha, Godoy, \& Ambiel, 2010).

Os interesses profissionais são amplamente investigados na literatura científica pela sua capacidade preditiva de escolhas uma vez que guiam comportamentos de indivíduos de acordo com o ambiente e com os objetivos pessoais (Rounds \& Su, 2014). Além disso, permitem o engajamento nas atividades referentes à escolha profissional que podem ou não ser concretizadas (Ambiel \& Noronha, 2012). Entre os teóricos que realizaram conceitualização dos interesses profissionais, destaca-se Holland (1975) que, na tentativa de responder as indagações sobre os processos de escolha e as variáveis nestes imbricadas, desenvolveu a Teoria de Personalidade Vocacional e Ambientes de Trabalho.

O autor definiu seis tipos de personalidade vocacionais e seis modelos ambientais: Realista, Investigativo, Artístico, Social, Empreendedor e Convencional. O tipo Realista é introvertido e tem pouca habilidade interpessoal, tendo boa coordenação motora e agilidade, preferindo lidar com questões concretas. O tipo Investigativo é analítico, crítico e introvertido, apresentando aptidões para lidar com o manuseio de palavras e ideias. O tipo Artístico, por sua vez, gosta de contato interpessoal, valoriza os vínculos humanos e tem facilidade para se expressar, demonstrando abertura aos estímulos subjetivos e emocionais. O tipo Social tende a ser sensível, humanista, responsável, com habilidade verbal e com uma necessidade maior interação social. O tipo Empreendedor apresenta-se como aventureiro, impulsivo e extrovertido, demonstrando capacidades verbais e persuasivas, e um interesse por temas relacionados a economia, poder e liderança. E por fim, o tipo Convencional que tem por características a persistência, criatividade e obediência, mostrando-se eficiente em tarefas que demandam organização e metas (Holland, 1975).

A partir de suas descrições sobre as tipologias, foi desenvolvido o Self-Directed Search (SDS) (Holland, 1975), instrumento amplamente utilizado em processos de orientação de adolescentes e adultos, traduzido e adaptado para mais de 16 idiomas (Foutch, McHugh, Bertoch, \& Reardon, 2014). A versão brasileira do instrumento (Primi, Mansão, Muniz, \& Nunes, 2010) tem sido largamente utilizada para a produção de trabalhos no âmbito nacional (Magalhães, 2013; Noronha, Mansão, Silva, Freitas, \& Pereira 2013; Sartori, Noronha, \& Nunes, 2009), investigando variáveis de processo de tomada de decisão, aconselhamento e construção de carreira.

Além dos estudos de propriedades psicométricas ou de relação com instrumentos de interesses, encontram-se pesquisas que verificaram correlações entre $O$ SDS com instrumentos que mensuram outras variáveis latentes, como inclinação para a escolha (Mansão, Noronha, \& Otatti, 2011; Okino \& Pasian, 2015), habilidades cognitivas (Nunes \& Noronha, 2009b) afetos e personalidade (Barros, Noronha, \& Ambiel, 2015) e autoeficácia (Nunes \& Noronha, 2009a). Evidencia-se, portanto, a expressividade do SDS em estudos empíricos bem como sua aplicabilidade em processos de Orientação Profissional e de Carreira como facilitador da verificação de interesses profissionais.

Nessa direção, a utilização de instrumentos de interesses profissionais dentro dos processos de OPC auxilia o orientando a clarificar suas inclinações, promovendo o autoconhecimento e diminuindo as dificuldades para a tomada de decisão. Apesar de todos os meios e métodos, encontram-se indivíduos com dificuldades para tomar uma decisão profissional por fatores diversos e em diferentes graus de complexidade. Tais dificuldades podem estar relacionadas com o grau de maturidade (Proyer, Sidler, Weber, \& Ruch, 2012) ou com a falta de clareza dos adolescentes sobre a multiplicidade de profissões e áreas de estudo que podem causar conflitos relacionados à escolha (Campos \& Noronha, 2016; Melo-Silva, Lassance, \& Soares, 2004). Verifica-se ainda que experiências de conflitos familiares influenciam diretamente no nível de indecisão que também podem ser mais acentuadas em filhos cujo os pais não possuem curso de graduação (Choi et al., 2011; Stoltz \& Young, 2012) ou que estão em situação de desemprego (Faria, 2013; Magalhães, Alvarenga, \& Teixeira, 2012).

Os impactos que as práticas educativas dos pais exercem sobre o desenvolvimento dos filhos têm sido alvo de investigações em OPC (Teixeira, Bardagi, \& Gomes, 2004; Teixeira, Oliveira, \& Wottrich, 2006). Os comportamentos são agrupados de maneira a definir "tipos" ou estilos parentais, sendo que duas principais práticas educativas têm sido descritas como exigência e responsividade. Exigência é uma dimensão caracterizada por atitudes controladoras e estabelecimento de regras e limites. Responsividade está ligada a comportamentos afetivos, incentivos dos pais à 
autonomia de seus filhos e prevalência de diálogo nas relações pais-filhos. Essas duas dimensões, quando combinadas, caracterizam quatro estilos parentais: autoritário, autoritativo, indulgente e negligente (Teixeira et al., 2006). Filhos educados com alto nível de exigência possuem bom desempenho e competência, obediência e menores índices de problemas comportamentais. Porém, altos níveis de exigência também podem desencadear nos indivíduos preocupação excessiva, baixa autoestima e transtornos psicológicos. Enquanto a responsividade relaciona-se a indivíduos mais autoconfiantes, com boa autoestima e bem-estar psicológico (Teixeira et al., 2004).

A esse respeito, Teixeira et al. (2004) desenvolveram a Escala de Responsividade e Exigência (ERE), que visa avaliar as dimensões de responsividade e exigências parentais de adolescentes. O instrumento foi elaborado buscando refinar uma escala anteriormente testada no Brasil (Costa, Teixeira, \& Gomes, 2000) e, para tanto, foram desenvolvidos novos itens para melhorar a fidedignidade da escala. Desde então, a ERE vem sendo utilizada em estudos que visam avaliar a influência dos estilos parentais em contextos como a relação com as práticas educativas (Pacheco, Silveira, \& Schneider, 2008), a instabilidade de metas (Magalhães, Alvarenga, \& Teixeira, 2012) e a tomada de decisões de carreiras (Oliveira \& Dias, 2013).

Desse modo, a compreensão da influência familiar na construção da trajetória profissional é de fundamental importância, uma vez que os indivíduos desenvolvem seus papéis sociais a partir desse núcleo (Savickas, 2005). $\mathrm{Na}$ visão dos genitores, estes percebem que contribuem para o desenvolvimento de carreira dos filhos por meio do diálogo, oferecendo informações sobre as profissões e mercado de trabalho, bem como com a sua própria experiência profissional e que essa influência pode ter impactos positivos e negativos nas escolhas dos filhos (Oliveira \& Dias, 2013). Essas percepções também são corroboradas pelos filhos que entendem que uma influência positiva dos genitores tende a incentivar a autonomia e prazer nos estudos, ocasionando sucesso na escolha (Carvalho \& Taveira, 2009).

A autonomia proporciona ao jovem o sentimento de segurança e atitudes positivas frente à carreira, minimizando a indecisão na escolha profissional (Campos \& Noronha, 2016). Utilizando o Inventário de Dificuldades de Decisão Profissional (IDDP) e o Revised Life Orientation Test Brasil (LOT-R Brasil) em jovens aprendizes e estudantes do ensino médio/técnico, as autoras encontraram correlações negativas de baixa magnitude entre os fatores referentes à indecisão profissional e otimismo, enquanto que indecisão profissional apareceu mais expressivamente em indivíduos pessimistas e com maiores pontuações em Imaturidade para a escolha. Assim, jovens mais seguros tendem a apresentar maior êxito na escolha de uma profissão da mesma forma que jovens imaturos são mais pessimistas em relação ao seu futuro profissional resultando em maiores dificuldades em escolher uma ocupação.

Também utilizando o IDDP, Ambiel, Noronha e Santos (2011) relacionaram as dificuldades de decisão com a autoeficácia para escolha profissional e os resultados indicaram, ainda que com correlações fracas, que indivíduos com menores crenças de autoeficácia tendem a apresentar maior insegurança e imaturidade para a escolha. Importante salientar que as crenças de autoeficácia têm como uma das fontes de origem a aprendizagem vicária que é decorrente da observação de outros indivíduos executando tarefas e a comparação do seu próprio nível de capacidade frente aos seus semelhantes (Bandura, 1977). Nesse sentido, novamente os pais, enquanto primeiro núcleo social, apresentam-se como modelos que impactam o desenvolvimento das crenças de autoeficácia e, por consequência, o nível de segurança e maturidade para a escolha por meio da aprendizagem vicária.

Acerca da maturidade como um fator relacionado com a decisão profissional, Primi et al. (2001), correlacionaram o SDS com o IDDP e, com o Inventário Fatorial de Personalidade (IFP). Os autores se fundamentaram nas expectativas de que encontrariam associações entre indiferenciação de características de personalidade e dificuldade de decisão. Entretanto, os dados encontrados permitiram confirmar, somente de modo parcial, que tal hipótese era correta, uma vez que, tanto no IFP quanto no SDS, não foram detectadas correlações expressivas entre o IDDP e perfis menos diferenciados. $\mathrm{O}$ aspecto do IDDP que aponta imaturidade demonstrou correlações positivas com perfis com menor diferenciação do SDS, o que permite dizer que indivíduos menos maduros para decisão profissional apresentam perfis de interesses menos coerentes.

Nota-se que estudos voltados para a compreensão da relação entre as variáveis interesses profissionais, indecisão profissional e a influência familiar tem sido pautados em pesquisas. Compreender se existem relações entre as variáveis interesses e decisões profissionais e estilos parentais podem contribuir para o desenvolvimento de estudos e intervenções futuras voltadas às influências existentes na relação entre pais e filhos. Com 
intuito de avançar as discussões na área e, aumentar o entendimento da influência parental na constituição dos interesses e no processo de tomada de decisão, este estudo tem como objetivo verificar as associações entre interesses profissionais, dificuldade de decisão profissional e estilos parentais, bem como identificar possíveis diferenças em função do sexo e da idade.

\section{Método}

\section{Participantes}

Participaram desta pesquisa 473 adolescentes, sendo $66 \%$ do sexo feminino $(n=312)$ e $34 \%$ do sexo masculino $(n=161)$, com idades entre 14 e 19 anos $(M=16,55 ; D P=0,78)$. Todos os participantes foram oriundos de escolas públicas de cidades de pequeno porte do interior da região oeste do estado de São Paulo e cursavam o ensino médio distribuídos entre as três séries.

\section{Instrumentos}

a) Self-Directed Search Carrer Explorer (SDS) (Holland, 1975, versão adaptada Primi et al., 2010): o instrumento é destinado para verificação de interesses profissionais, estruturado em quatro seções: atividades, competências, carreiras e habilidades. As seções incluem itens que abordam os seis tipos propostos por Holland: Realista (R), Investigador (I), Artístico (A), Social (S), Empreendedor (E) e Convencional $(\mathrm{C})$. As respostas para as seções atividades, competências e carreiras são dicotômicas - S (sim) ou N (não), compostas por 66 itens cada. As respostas da seção Habilidades são de tipo Likert de sete pontos, variando de 1 (baixa habilidade) até 7 (alta habilidade) sendo essa seção composta por 12 itens. Em relação à precisão, o instrumento brasileiro apresentou índices satisfatórios obtendo coeficientes alfa de Cronbach entre 0,87 (Realista) e 0,90 (Convencional).

b) Inventário de Levantamento das Dificuldades de Decisão Profissional (IDDP) - (Primi, et al., 2000): o instrumento tem por objetivo verificar dificuldades para tomada de decisão profissional. O IDDP é composto por 81 itens que formaram 17 fatores, agrupados quatro fatores de segunda ordem, sendo: Fator 1 - Insegurança e falta de informação para tomada de decisão $(\alpha=0,89)$; Fator 2 - Busca de prestígio social das profissões e retorno financeiro $(\alpha=0,79)$; Fator 3 - Imaturidade para escolha associado à falta de motivação para a tomada de decisão $(\alpha=0,70)$; Fator 4 - Conflitos com pessoas significativas $(\alpha=0,71)$. O instrumento é respondido em escala do tipo Likert de sete pontos variando entre 1 (conteúdo totalmente contrário ao que você pensa) e 7 (conteúdo totalmente de acordo com o que vocêpensa).

\section{c) Escala de Responsividade e Exigência (ERE)}

(Teixeira et al., 2004): o instrumento tem como objetivo avaliar níveis de exigência e responsividade parentais percebidas entre adolescentes. A escala é composta por 24 itens, respondidos em escala Likert de cinco pontos variando entre 1 (ocorrem com pouca frequência) a 5 (ocorrem com muita frequência). Análises de componentes principais indicaram a existência dos dois fatores principais e com índices de precisão (alfa de Cronbach) de 0,81 para o fator Exigência e 0,91 para o fator Responsividade.

\section{Procedimentos}

O projeto foi autorizado pelo Comitê de Ética em Pesquisas da Universidade O projeto foi autorizado pelo Comitê de Ética em Pesquisas da Universidade do Oeste Paulista (CAAE: 30888314.0.0000.5515). As coletas ocorreram entre os meses de março a novembro do ano de 2016 em workshops de Orientação Profissional oferecidos para alunos de escolas do interior de São Paulo. Para a recolha dos dados, os pais assinaram previamente o Termo de Consentimento Livre e Esclarecido e os adolescentes assinaram o Termo Assentimento Livre e Esclarecido. A aplicação dos instrumentos ocorreu de forma coletiva em dias e horários previamente agendados para o workshop na universidade.

\section{Análise de Dados}

Os dados foram analisados por meio do software Statistical Package for the Social Sciences (SPSS) versão 22 com estatísticas descritivas para caracterização da amostra. Posteriormente, ocorreram as estatísticas inferenciais com teste $t$ de Student para diferenciação entre grupos, sendo que foram considerados os resultados com grau de significância estatística $(p<0,05)$. Além disso, foram realizadas análises de correlação de Pearson entre os fatores dos instrumentos. Foram utilizados os parâmetros estabelecidos por Dancey \& Reid (2013) para interpretação das correlações, sendo elas consideradas fracas ( $r$ entre 0,0 a 0,30$)$, moderadas $(r$ entre 0,40 a 0,60$)$ e fortes ( $r$ entre 0,70 a 1$)$.

Psico-USF, Bragança Paulista, v. 23, n. 4, p. 693-703, out./ dez. 2018 


\section{Resultados}

Inicialmente foram calculadas as médias e desvios padrões das respostas aos instrumentos, sendo que no SDS as maiores médias foram obtidas para a tipologia Convencional $(M=66,88 ; D P=6,07)$ e a menor para a tipologia Social $(M=60,76 ; D P=9,66)$. Por sua vez, no IDDP a maior média foi alcançada para o fator Insegurança e falta de informação para tomada de decisão $(M=110,42 ; D P=27,93)$ e a menor média para o fator Imaturidade para escolha associado e falta de motivação para a tomada de decisão $(M=22,10 ; D P=8,65)$. Em relação à ERE, as maiores médias foram obtidas para Exigência Materna $(M=48,16$; $D P=13,62)$ e Responsividade Paterna $(M=42,69 ; D P=14,94)$ e as menores para Exigência Paterna $(M=41,32 ; D P=13,62)$ e Responsividade Materna $(M=41,32 ; D P=11,18)$.

$\mathrm{Na}$ sequência, realizou-se o teste $t$ de Student, buscando diferenças nas médias em função da variável sexo e idade, sendo que para esta última variável a amostra foi dividida entre participantes com idade até 16 anos $(N=232)$ e a partir de $17 \operatorname{anos}(N=240)$. A decisão por essa divisão ocorreu em função da idade média dos participantes do estudo $(M=16,55)$ que permitiu gerar dois grupos equilibrados em termos de quantidade de participantes. A Tabela 1 apresenta os resultados que obtiveram significância estatística.

Considerando a variável sexo, foram observadas diferenças estatisticamente significativas. Em relação aos interesses profissionais (SDS), mulheres obtiveram maiores médias em relação aos homens nas tipologias Realista e Empreendedor. Por outro lado, os homens obtiveram maiores médias do que mulheres na tipologia Social. Já no IDDP foram encontradas diferenças apenas no fator Imaturidade para escolha associado à falta de motivação para a tomada de decisão, no qual homens obtiveram maiores médias quando comparados às mulheres. No que se refere aos estilos parentais, apenas para o fator Exigência Materna, verificaram-se diferenças com mulheres tendo maiores pontuações do que os homens.

Nas comparações por grupos de idade, não foram encontradas diferenças significativas nas tipologias do SDS e nem nos fatores de Responsividade e Exigência (ERE). Nos fatores do IDDP houve diferenciação com significância estatística, sendo que os participantes com idade a partir de 17 anos apresentaram maiores médias no fator Insegurança e falta de informação para tomada de decisão quando comparados com os participantes mais novos. $\mathrm{Na}$ sequência foram realizadas correlações de Pearson entre os fatores do SDS, IDDP e ERE, sendo que os resultados são apresentados na Tabela 2.

Utilizando os critérios de Dancey e Reid (2013) para interpretação dos coeficientes de correlações, observam-se na Tabela 2 que as correlações entre os fatores do SDS foram significativas estatisticamente, em sentido positivo e variaram de intensidade fraca à

Tabela 1

Comparações de Médias em Função do Sexo e Idade dos Participantes

\begin{tabular}{lccccc}
\hline Fator & Variável & Média & Desvio padrão & $t$ & $p$ \\
\hline Realista (SDS) & M & 63,88 & 6,57 & 3,95 & 0,00 \\
& F & 66,99 & 10,41 & & \\
Social (SDS) & M & 62,36 & 9,39 & $-2,62$ & 0,01 \\
& F & 59,93 & 9,71 & & \\
Empreendedor (SDS) & M & 60,19 & 6,23 & 2,47 & 0,01 \\
Imaturidade para escolha (IDDP) & F & 61,70 & 6,23 & & \\
& M & 23,80 & 9,09 & $-2,98$ & 0,00 \\
Exigência Materna (ERE) & F & 21,22 & 8,29 & & 0,01 \\
Insegurança e falta de informação (IDDP) & M & 46,70 & 10,00 & 2,37 & 0 \\
& F & 48,92 & 8,91 & & \\
\end{tabular}

Nota. $\mathrm{M}=$ Masculino; $\mathrm{F}=$ Feminino; $\leq 16$ = idade igual ou menor que 16 anos; $\geq 17=$ idade igual ou maior que 17 anos. 
Tabela 2

Correlaçôes entre os Fatores do SDS, IDDP e ERE

\begin{tabular}{|c|c|c|c|c|c|c|c|c|c|c|c|c|c|c|}
\hline & 1 & 2 & 3 & 4 & 5 & 6 & 7 & 8 & 9 & 10 & 11 & 12 & 13 & 14 \\
\hline 1 & 1 & & & & & & & & & & & & & \\
\hline 2 & $0,17^{* *}$ & 1 & & & & & & & & & & & & \\
\hline 3 & $0,30^{* *}$ & $0,24^{* *}$ & 1 & & & & & & & & & & & \\
\hline 4 & $0,24^{* *}$ & $0,20^{* *}$ & $0,24^{* *}$ & 1 & & & & & & & & & & \\
\hline 5 & $0,17^{* *}$ & $0,17^{* *}$ & $0,30^{* *}$ & $0,26^{* *}$ & 1 & & & & & & & & & \\
\hline 6 & $0,27^{* *}$ & $0,16^{* *}$ & $0,22^{* *}$ & $0,17^{* *}$ & $0,47^{* *}$ & 1 & & & & & & & & \\
\hline 7 & 0,26 & 0,05 & 0,07 & $-0,02$ & 0,02 & $-0,00$ & 1 & & & & & & & \\
\hline 8 & $-0,06$ & $-0,01$ & 0,00 & $-0,03$ & $-0,01$ & $-0,02$ & $0,44^{* *}$ & 1 & & & & & & \\
\hline 9 & $-0,04$ & 0,03 & $-0,02$ & $-0,04$ & $-0,07$ & $-0,02$ & $0,43^{* *}$ & $0,23^{* *}$ & 1 & & & & & \\
\hline 10 & $-0,10$ & 0,02 & $-0,06$ & $-0,04$ & $-0,10^{*}$ & $-0,07$ & $0,27^{* *}$ & $0,49^{* *}$ & $0,68^{* *}$ & 1 & & & & \\
\hline 11 & $-0,00$ & $-0,00$ & $-0,00$ & $-0,01$ & $-0,01$ & $-0,06$ & 0,03 & $-0,13^{* *}$ & 0,02 & $-0,03$ & 1 & & & \\
\hline 12 & 0,01 & 0,00 & 0,01 & $-0,01$ & $-0,14^{*}$ & $-0,04$ & $0,19^{*}$ & $0,20^{* *}$ & $0,22^{* *}$ & $0,22^{* *}$ & $0,32^{* *}$ & 1 & & \\
\hline 13 & $-0,07$ & $-0,00$ & 0,00 & 0,00 & 0,01 & $-0,04$ & $-0,06$ & $-0,15^{* *}$ & $-0,03$ & $-0,04$ & $0,55^{* *}$ & $0,13^{* *}$ & 1 & \\
\hline 14 & 0,08 & 0,03 & 0,03 & $-0,02$ & 0,06 & 0,02 & 0,07 & $-0,12^{* *}$ & 0,06 & $-0,07$ & $0,51^{* *}$ & $0,35^{* *}$ & $0,37^{* *}$ & 1 \\
\hline
\end{tabular}

Nota. $*_{p}<0,05 ; *$; $<<0,001$; 1- Realista (SDS); 2- Investigativo (SDS); 3- Artístico (SDS); 4- Social (SDS); 5- Empreendedor (SDS); 6- Convencional (SDS); 7; Exigência Materna (ERE); 8- Responsividade Materna (ERE); 9- Exigência Paterna (ERE); 10- Responsividade Paterna (ERE); 11- Insegurança e falta de Informação para tomada de decisão; 12- Busca de prestígio social das profissões e retorno financeiro; 13- Imaturidade para escolha associado à falta de motivação para a tomada de decisão; 14- Conflitos com pessoas significativas.

moderada. Em relação aos coeficientes de correlação entre os fatores da ERE, observou-se que estas foram significativas, em direção positiva e com magnitudes de valores entre fracos a moderados. Por fim, o mesmo também ocorreu para as correlações entre os fatores do IDDP que foram estatisticamente significativas, em direção positiva e com magnitude variando entre fracas e moderadas.

$\mathrm{Na}$ comparação entre os fatores dos dois instrumentos SDS e IDDP, apenas a tipologia Empreendedor e o fator Busca de prestígio social das profissões e retorno financeiro obtiveram uma correlação negativa, com significância estatística e de intensidade fraca. As demais correlações entre os instrumentos foram nulas ou sem significância estatística. Ao analisar as correlações entre as tipologias do SDS com os fatores da ERE, verifica-se que apenas o fator Responsividade Paterna obteve correlações significativas estatisticamente com as tipologias Realista e Empreendedor em sentido negativo e com magnitude fraca.

Por fim, em relação às correlações obtidas entre os fatores do IDDP e da ERE, nota-se que a maioria das correlações foram significativas estatisticamente com magnitude fraca à moderada. O fator Busca de prestígio social das profissões e retorno financeiro correlacionou-se positivamente com as dimensões Exigência Materna, Exigência Paterna e Responsividade Paterna. Ao considerar o fator Responsividade Materna todas as correlações foram significativas com as dimensões do IDDP e a maioria em sentido negativo.

\section{Discussão}

Considerando os resultados apresentados, percebe-se que o objetivo deste trabalho foi atingido ao verificar as associações entre interesses profissionais, dificuldade de decisão profissional e estilos parentais, bem como identificar possíveis diferenças em função do sexo e da idade. Estudos nessa perspectiva são necessários ao considerar que a escolha profissional requer uma tomada de decisão complexa, envolvendo diversos fatores pessoais e profissionais (Melo-Silva et al., 2004), bem como pelas constantes transformações sociais, econômicas e políticas que afetam inúmeros setores sociais (Duarte, 2009), inclusive no âmbito familiar. Tais transformações implicam em avanços para o entendimento das temáticas relacionadas à construção de carreira (Duarte, 2015). 
Inicialmente em relação às diferenças encontradas nas pontuações dos participantes nos instrumentos, cabe destacar o resultado da comparação entre os sexos nos interesses profissionais. Neste estudo, as mulheres obtiveram maiores médias em tipologias que agregam ocupações consideradas tradicionalmente como masculinas, quais sejam, as tipologias Realista e Empreendedor, e os homens na tipologia Social com ocupações em geral realizadas por mulheres (Holland, 1975). Embora o resultado divirja de outras pesquisas que utilizaram o SDS (Armstrong, Su, \& Rounds, 2011; Mansão \& Yoshida, 2006; Su, Rounds, \& Armstrong, 2009), tal dado parece indicar um progresso em relação à equidade dos gêneros frente ao mundo do trabalho e a conquista de espaços muitas vezes restritos por estigmas sociais impostos aos homens e mulheres.

Nessa direção, reforçam-se os apontamentos de Duarte (2015) e Savickas et al. (2009) de que as realidades do século XXI são diferentes das realidades históricas que subsidiaram o desenvolvimento de modelos e paradigmas durante séculos anteriores, cabendo a necessidade de atualizações na compreensão do desenvolvimento de carreira, uma vez que a realidade é outra, permitindo que novos papéis sejam desenvolvidos e espaços ocupados. Tal constatação reflete ainda na necessidade de aperfeiçoar os instrumentos de modo a minimizar os vieses de gênero nas medidas de interesses, possibilitando que o uso maximize as oportunidades de carreira com a exploração de áreas sem restrição de gênero (Armstrong, Su, \& Rounds, 2011).

Outra diferença encontrada quanto ao sexo ocorreu nas pontuações da ERE, sendo que as adolescentes pontuaram mais em Exigência materna quando comparada aos participantes do sexo masculino. Assim, as adolescentes percebem a figura materna como mais controladora e que estabelecem normas e metas de comportamento, fato que também está relacionado ao contexto social imposto ao papel da mulher como a responsável pela educação dos filhos. Resultados semelhantes foram encontrados por Weber, Prado, Viezzer e Brandenburg (2004), indicando que as meninas são vistas como mais frágeis, demandando de maior controle e cuidado, enquanto os meninos são vistos como mais fortes e autônomos, reflexos de uma cultura machista que demanda de desconstrução acerca dos gêneros.

A idade mostrou-se como uma variável que influenciou apenas as respostas no IDDP, uma vez que os participantes mais velhos apresentaram maiores médias no fator Insegurança e falta de informação para tomada de decisão. Apesar de parecer contraditório, o resultado corresponde com aspectos relacionados à faixa etária. Os adolescentes, em período de conclusão do ensino médio, farão escolhas como ingressar no mercado de trabalho ou iniciar um curso superior, o que pode gerar insegurança e dificulta a tomada de decisão. Tal dado reforça ainda mais a necessidade de intervenções em OPC que possibilitem ao jovem explorar o mundo das profissões e do trabalho, diminuindo suas angústias e permitindo tomadas de decisões concisas ao projeto de carreira (Almeida, Magalhães, \& Féres-Carneiro, 2014).

No que se refere às correlações entre os instrumentos, quando correlacionados, os fatores do IDDP com o SDS houve apenas uma correlação com significância estatística e em sentido negativo entre a tipologia Empreendedor e o fator Busca de prestígio social das profissões e retorno financeiro. Esse resultado é coerente com o pressuposto por Holland (1975) sobre as características das pessoas do tipo Empreendedor que tendem a valorizar o exibicionismo, aspectos econômicos e estéticos e persuadir pessoas e liderar equipes. Assim, os adolescentes que apresentam maiores dificuldades em buscar prestígio por meio da ocupação ou que estas possam gerar conflitos com outras pessoas, tendem a apresentar menores pontuações em interesses empreendedores.

Por sua vez, na comparação dos tipos de interesses com os estilos parentais, apenas o fator Responsividade Paterna obteve correlações significativas estatisticamente com as tipologias Realista e Empreendedor e em sentido negativo. Esse dado sugere que quanto mais os adolescentes percebem seus genitores masculinos como menos responsivos mais tendem a ter interesses voltados para ocupações realistas e empreendedoras. Nesse sentido, ao considerar que a Responsividade é a capacidade dos pais de propiciar o desenvolvimento da autoafirmação, da autonomia e da afetividade (Costa et al., 2000), quando não ocorre esse processo, pode-se pensar que os filhos tendem a preferir trabalhar sozinhos, com atividades que não exijam expressões subjetivas e poucos sociáveis como no caso dos realistas (Holland \& Holland, 1977).

Nas correlações entre indecisão e estilos parentais, o fator Busca de prestígio social das profissões e retorno financeiro correlacionou-se positivamente com as dimensões Exigência Materna, Exigência Paterna e Responsividade Paterna. O resultado pode indicar que, quanto mais os genitores controlam os comportamentos por meio de normas ou por cobranças, mais baixos tendem a ser os níveis de autoestima dos filhos. Isto também fará com que os adolescentes tenham 
dificuldades em tomar decisões por ocupações de prestígio social. Nessa perspectiva, a Responsividade excessiva pode resultar em jovens inseguros e menos otimistas, por conseguinte, com maiores dificuldades para a tomada de decisão (Campos \& Noronha, 2016).

A dimensão Responsividade Materna apresentou correlações com todas as dimensões do IDDP. Os achados indicam que jovens com menores dificuldades para decisão em função da insegurança, falta de informação, imaturidade ou por conflitos com pessoas significativas tendem a ter a percepção de suas mães com maiores atitudes compreensivas e que permitem mais diálogo e apoio emocional. Pode-se pensar que essas mães adotam posturas como as apontadas por Oliveira e Dias (2013) como positivas no processo de construção de carreira, fornecendo informações sobre o mercado de trabalho e das profissões ou servindo como modelo profissional por meio da sua própria trajetória de carreira.

Assim, com base nos achados deste estudo, percebe-se que as figuras paternas e maternas apresentam grande influência no processo de tomada de decisão de carreira por parte dos adolescentes. Tal dado é um indicativo da necessidade de incluir as figuras parentais nos processos de Orientação Profissional e de Carreira com intuito de compreender a dinâmica da escolha do orientando e minimizar potenciais dificuldades para decisão, uma vez que, esta é influenciada por inúmeros aspectos ambientais, sociais e econômicos (Rounds \& Su, 2014).

O estudo contribui com avanços sobre o entendimento das variáveis para a área de Orientação Profissional e de Carreira, em especial no que diz respeito aos propósitos iniciais, ou seja, a verificação de associações entre os interesses profissionais e as variáveis indecisão e estilos parentais. Cabe ressaltar os principais achados que apontaram correlação negativa de fraca magnitude e intensidade entre a tipologia Empreendedor (SDS) e o fator Busca de prestígio social das profissões e retorno financeiro (IDDP), correlações em sentido negativo e com magnitude fraca entre as tipologias Realista e Empreendedor (SDS) e o fator Responsividade Paterna (ERE) Também foram encontradas correlações positivas entre o fator Busca de prestígio social das profissões e retorno financeiro (IDDP) com as dimensões Exigência Materna, Exigência Paterna e Responsividade Paterna (ERE).

Pode-se inferir que, embora a maioria das correlações tenham sido de magnitude fraca à moderada, existem associações entre os construtos interesses, indecisão e estilos parentais que não devem ser desprezadas. Assim, sugere-se a realização de novas pesquisas com populações de estados diferentes e incluindo também alunos de escolas particulares com intuito de verificar diferenças em função das características sociodemográficas. Outro apontamento para pesquisas futuras seria a inserção dos pais como respondentes de escalas que revelem suas próprias percepções acerca de seus estilos educativos, o que poderá fornecer novos subsídios para o entendimento da estreita associação anunciada por este estudo, entre tais estilos e as escolhas de carreira de adolescentes e jovens. Estudos com figuras parentais e filhos, permitiriam verificar se há congruência entre as percepções e qual a influência da trajetória profissionais dos pais no processo de construção de carreira dos filhos.

Como limitação do estudo pode ser destacada a falta de informações sociodemográfias dos pais (idade, profissão, escolaridade). Tais informações certamente facilitariam o diálogo com os referenciais teóricos, especialmente em relação às possíveis interferências dos pais na formação dos interesses e inclinação para determinadas tipologias. Além disso, é preciso ressaltar que a amostra é restrita a uma região de um único estado brasileiro, podendo refletir apenas a realidade local.

Apesar da ampla produção abordando os interesses profissionais, os estudos são escassos quando se busca compreender a influência parental na constituição dos interesses e nos processos de tomada de decisão. Nessa instância, encontra-se a originalidade do presente estudo, bem como as expectativas de que com novas investigações sobre o tema, vislumbrem-se propostas interventivas em OPC nas quais sejam clarificados de forma eficiente os impactos das práticas educativas parentais na configuração dos interesses profissionais de adolescentes.

\section{Referências}

Ambiel, R. A. M., Noronha, A. P. P., \& Santos, A. A. A. dos. (2011). Escala de Autoeficácia para Escolha Profissional: avaliação preliminar das propriedades psicométricas. Psicologia para América Latina, (21), 57-71. Recuperado de http://pepsic.bvsalud.org/ pdf/psilat/n21/a05.pdf

Ambiel, R. A. M., \& Noronha, A. P. P. (2012). Autoeficácia para Escolha Profissional: Teoria, Pesquisas e Avaliação. Psicologia em Pesquisa, 6(2), 171-178. doi: 10.5327/Z1982-12472012000200010

Psico-USF, Bragança Paulista, v. 23, n. 4, p. 693-703, out./ dez. 2018 
Armstrong, P. I., Su, R., Rounds, J. (2011). Vocational interests: The road less traveled. In ChamorroPremuzic, T., Von Strumm, S., Furnham, A. (Eds.), Handbook of individual differences (pp. 608-631). Oxford: Wiley-Blackwell.

Bacal, M. E. A., Magalhães, A. S., \& Féres-Carneiro, T. (2014). Transmissão geracional da profissão na familia: repetição e diferenciação. Psico, 45(4), 454462. doi: 10.15448/1980-8623.2014.4.15344

Barros, M. V. C., Noronha, A. P. P., \& Ambiel, R. A. M. (2015). Afetos, interesses profissionais e personalidade em alunos do ensino médio. Revista Brasileira de Orientação Profissional, 16(2), 161-171. Recuperado de http://pepsic.bvsalud.org/pdf/ rbop/v16n2/07.pdf

Bandura, A. (1977). Self-efficacy: Toward a unifying theory of behavioral change. Psychological Review, 84(2), 191-215. doi:10.1037/0033-295X.84.2.191

Carvalho, M., \& Taveira, M. C. (2009). Influência de pais nas escolhas de carreira dos filhos: visão de diferentes atores. Revista Brasileira de Orientação Profissional, 10(2), 33-41. Recuperado de http://pepsic. bvsalud.org/pdf/rbop/v10n2/v10n2a05.pdf

Campos, R. R. F., \& Noronha, A. P. P. (2016). A relação entre indecisão profissional e otimismo disposicional em adolescentes. Temas em Psicologia, 24(1), 219-232. doi: 10.9788/TP2016.1-15

Choi, B. Y., Park, H., Yang, E., Ki Lee, S., Lee, Y., \& Min Lee, S. (2011). Understanding career decision self-eficacy: A meta-analytic approach. Journal of Career Development, 39(5),443-460. doi: $10.1177 / 0894845311398042$.

Costa, F. T., Teixeira, M. A. P., \& Gomes, W. B. (2000). Responsividade e exigência: Duas escalas para avaliar estilos parentais. Psicologia: Reflexão e Crítica, 13(3), 465-473. Recuperado em http://www.scielo. $\mathrm{br} / \mathrm{pdf} / \mathrm{prc} / \mathrm{v} 13 \mathrm{n} 3 / \mathrm{v} 13 \mathrm{n} 3 \mathrm{a} 14$

Dancey, C. P., \& Reid, J. (2013). Estatística sem matemática para psicologia. Porto Alegre: Artmed.

Duarte, M. E. (2009). Um século depois de Parsons: Escolher uma profissão ou apostar na psicologia da construção da vida? Revista Brasileira de Orientação Profissional, 10(2), 5-14. Recuperado de http:// www.redalyc.org/pdf/2030/203014923003.pdf

Duarte, M. E. (2015). Inovação em orientação e aconselhamento de carreira: Mitos e realidades. Revista
Brasileira de Orientação Profissional, 16(2), 110-121. Recuperado de http://pepsic.bvsalud.org/pdf/ rbop/v16n2/03.pdf

Faria, L. C. (2013). Influência da condição de emprego/desemprego dos pais na exploração e indecisão vocacional dos adolescentes. Psicologia: Reflexão \& Crítica, 26(4), 772-779. doi: 10.1590/ S0102-79722013000400018

Foutch, H., McHugh, E. R., Bertoch, S. C., \& Reardon, R. C. (2014). Creating and Using a Database on Holland's Theory and Practical Tools. Journal of Career Assessment, 22(1), 188-202. doi: $10.1177 / 1069072713492947$

Holland, J. L. (1975). Técnica de la elección vocacional: Tipos de personalidade y modelos ambientales (R. D. Guerrero, Trad.). México: Trillas.

Holland, J. L., \& Holland, J. E. (1977). Vocational indecision: More evidence and speculation. Journal of Counseling Psychology, 24(5), 404-414. doi: 10.1037/0022-0167.24.5.404

Magalhães, M. O. (2013). Validade de critério da Escala de Atividades do Questionário de Busca Autodirigida. Avaliação Psicológica, 12(1), 91-99. Recuperado de http://pepsic.bvsalud.org/pdf/avp/v12n1/ v12n1a12.pdf

Magalhães, M. O., Alvarenga, P., \& Teixeira, M. A.P. (2012). Relação entre estilos parentais, instabilidade de metas e indecisão vocacional em adolescentes. Revista Brasileira de Orientação Profissional, 13(1), 15-25. Recuperado de http://pepsic.bvsalud.org/ pdf/rbop/v13n1/04.pdf

Mansão, C. S. M., \& Yoshida, E. M. P. (2006). SDS questionário de busca autodirigida: Precisão e validade. Revista Brasileira de Orientação Profissional, 7(2), 67-79. Recuperado de http://pepsic.bvsalud. org/pdf/rbop/v7n2/v7n2a07.pdf

Mansão, C. S. M., Noronha, A. P. P., \& Ottati, F. (2011). Interesses profissionais: Análise correlacional entre dois instrumentos de avaliação. Revista Brasileira de Orientação Profissional, 12(2), 175-184. Recuperado de http://pepsic.bvsalud.org/pdf/ rbop/v12n2/05.pdf

Melo-Silva, L., Lassance, M. C. P., \& Soares, D. H. P. (2004). A Orientação Profissional no Contexto da Educação e Trabalho. Revista Brasileira de Orientação 
Profissional, 5(2), 31-52. Recuperado de http://pepsic.bvsalud.org/pdf/rbop/v5n2/v5n2a05.pdf

Noronha, A. P. P., Mansão, C. S. M., Silva, M. A., Freitas, P. C.S., \& Pereira, G. O. A. (2013). Evidências de validade convergente-discriminante para a avaliação dos tipos profissionais de Holland (ATPH). Psicologia: Ciência e Profissão, 33(1), 04-15. doi: 10.1590/S1414-98932013000100002

Nunes, M. F. O., \& Noronha, A. P. P. (2009a). Autoeficácia para atividades ocupacionais e interesses profissionais em estudantes do ensino médio. Psicologia: Ciência e Profissão, 29(1), 102-115. doi: 10.1590/S1414-98932009000100009

Nunes, M. F. O., \& Noronha, A. P. P. (2009b). Relações entre interesses, personalidade e habilidades cognitivas: Um estudo com adolescentes. PsicoUSF, 14(2), 131-141. Recuperado de http://pepsic. bvsalud.org/pdf/psicousf/v14n2/v14n2a02.pdf

Oliveira, C. T., \& Dias, A. C. G. (2013). Percepções parentais sobre sua participação no desenvolvimento profissional dos filhos universitários. Revista Brasileira de Orientação Profissional, 14(1), 61-72. Recuperado de http://pepsic.bvsalud.org/pdf/rbop/ v14n1/07.pdf

Okino, E. T. K., \& Pasian, S. R. (2015). Convergência entre interesses do SDS e inclinações motivacionais do BBT-BR no Brasil. Revista Brasileira de Orientaşão Profissional, 16(2), 137-148. Recuperado de http:// pepsic.bvsalud.org/pdf/rbop/v16n2/05.pdf

Pacheco, J. T. B., Silveira, L. M. B., \& Schneider, A. M. A. (2008). Estilos e práticas educativas parentais: Análise da relação desses construtos sob a perspectiva dos adolescentes. Psico, 39(1), 66-73. Recuperado de http://revistaseletronicas. pucrs.br/ojs/index.php/revistapsico/article/ view/1480/2797

Primi, R., Munhoz, M. A. H., Bighetti, C. A., Nucci, E. P. D., Pellegrini, M. C. K., \& Moggi, M. A. (2000). Desenvolvimento de um inventário de levantamento das dificuldades da decisão profissional. Psicologia: Reflexão e Crítica, 13(3), 451-453. doi: 10.1590/S0102-79722000000300013

Primi, R., Mansão, C. M., Muniz, M., \& Nunes, M. F. O. (2010). SDS-Questionário de Busca Autodirigida. Manual Técnico da versão brasileira. São Paulo: Casa do Psicólogo.
Primi, R., Pelegrini, M. C. K., Nucci, E. P., Bighetti, C. A., Munhoz, A. M. H., \& Moggi, M. A. (2001). Características de personalidade e indecisão profissional. Psico, 32(1), 81-96.

Proyer, R. T., Sidler, N., Weber, M., \& Ruch, W. (2012). A multi-method approach to studying the relationship between character strengths and vocational interests in adolescents. International Journal for Educational and Vocational Guidance, 12(2),141-157. doi:10.1007/s10775012-9223-x.

Rounds, J., \& Su, R. (2014). The Nature and Power of Interests. Current Directions in Psychological Science, 2323(2) 98-103. doi: 10.1177/0963721414522812

Sartori, F. A., Noronha, A. P.P., Godoy, S., \& Ambiel, R. A. M. (2010). Interesses profissionais de jovens de ensino médio: Estudo correlacional entre a Escala de Aconselhamento Profissional e o Self-Directed Search Carrier Explorer. Estudos em Psicologia (Campinas), 27(2). doi: 10.1590/S0103-166X2010000200009

Sartori, F. A., Noronha, A. P. P., \& Nunes, M. F. O. (2009). Comparações entre EAP e SDS: Interesses profissionais em alunos do ensino médio. Boletim de Psicologia, 59(130), 17-29. Recuperado de http://pepsic.bvsalud.org/pdf/bolpsi/v59n130/ v59n130a03.pdf

Savickas, M. L. (2005). The theory and practice of career construction. Em D. Brown \& R. Lent (Eds.), Career development and counselling: Putting theory and research to work (pp. 43-70). New York: John Wiley \& Sons.

Savickas, M. L., Nota, L., Rossier, J., Dauwalder, J. P., Duarte, M. E., Guichard, J., ... \& Van Vianen, A. E. (2009). Life designing: A paradigm for career construction in the 21st century. Journal of vocational behavior, 75(3), 239-250. doi: 10.1016/j. jvb.2009.04.004

Stoltz, K. B., \& Young, T. L. (2012). Applications of motivational interviewing in career counseling: Facilitating career transition. Journal of Career Development, 40, 1-18. doi:10.1177/0894845312455508

Su, R., Rounds, J., \& Armstrong, P. I. (2009). Men and things, women and people: A metaanalysis of gender and interests. Psychological Bulletin, 135(6), 859-884. doi: 10.1037/a0017364

Teixeira, M. A. P., Bardagi, M. P., \& Gomes, W. B. (2004). Refinamento de um instrumento para 
avaliar responsividade e exigência parental percebidas na adolescência. Avaliação Psicológica, 3(1), 1-12. Recuperado de http://pepsic.bvsalud.org/pdf/ avp/v3n1/v3n1a01.pdf

Teixeira, M. A. P., Oliveira, A. M., \& Wottrich, S. H. (2006). Escalas de Práticas Parentais (EPP): avaliando dimensões de práticas parentais em relação a adolescentes. Psicologia: Reflexão e Crítica, 19(3), 433-441. doi: 10.1590/S0102-79722006000300012
Weber, L. N. D., Prado, P. M., Viezzer, A. P., \& Brandenburg, O. J. (2004). Identificação de estilos parentais: $\mathrm{O}$ ponto de vista dos pais e dos filhos. Psicologia: Reflexão e Crítica, 17(3), 323-331. Recuperado de http://www.scielo.br/pdf/prc/v17n3/ a05v17n3.pdf

Recebido em: 03-01-2018

Reformulado em: 22-03-2018

Aprovado em: 03-05-2018

Sobre os autores:

Camélia Santina Murgo é psicóloga, doutora em Psicologia pelo Programa de Pós-graduação Stricto Sensu em Psicologia pela Pontifícia Universidade Católica de Campinas e docente do Programa de Mestrado em Educação da Universidade do Oeste Paulista.

E-mail: camélia@unoeste.br

Leonardo de Oliveira Barros é psicólogo, mestre e doutorando em Psicologia pelo Programa de Pós-Graduação Stricto Sensu em Psicologia da Universidade São Francisco (bolsista CAPES). (ÓRCID: 0000-0002-8406-0515).

E-mail: leonardobarros_lob@hotmail.com

Bárbara Cristina Soares Sena é psicóloga e mestranda em Educação pelo Programa de Pós-Graduação Stricto Sensu em Educação da Universidade do Oeste Paulista (bolsista CAPES).

E-mail: barbara.kristina08@gmail.com

Contato com os autores:

Camélia Santina Murgo

Rua Laguna 274, Apto. 610

Presidente Prudente-SP, Brasil

CEP: 19050-730 